Article

\title{
Non-Invasive Determination of Blood Glucose Concentration Using a Near-Field Sensor
}

\author{
Kseniya Zavyalova *, Aleksandr Gorst $\mathbb{D}$ and Aleksandr Mironchev \\ Department of Radiophysics, National Research Tomsk State University, 36 Lenin Ave., Tomsk 634050, Russia; \\ (A.G.); (A.M.) \\ * Correspondence: ksu.b@mail.ru
}

Received: date; Accepted: date; Published: date

\begin{abstract}
This article demonstrates a model of a near-field sensor, which is a combined slot antenna based on a flexible substrate RO3003. It is shown that the sensor has a high penetration of electromagnetic waves into highly absorbing media due to the length of the near field. Also it has a small size (diameter the sensor is $25 \mathrm{~mm}$, thickness $0.76 \mathrm{~mm}$ ). The simplified model of a hand simplified with a shallow vein depth was constructed. This model based on the experimentally obtained data of dielectric constant for glucose concentrations of $1.2-10 \mathrm{mmol} / \mathrm{l}$. The simulation results, carried out in the frequency range $0.5-5 \mathrm{GHz}$, showed the possibility of determining a small change in blood sugar level from the reflected signal data.
\end{abstract}

Keywords: glucose measurement; non-invasive; near-field; biosensor

\section{Introduction}

Diabetes mellitus has become a serious disease. It can cause many different diseases, including stroke. These deadly diseases are caused by the inability of the human body to produce and use insulin. It is a hormone that is essential for the processing of glucose in the blood. The number of people with diabetes is growing every year.

Back in 2002, Jones M. and Harrison J. M. described in their article [1] that the World Health Organization (WHO) expects an increase in the number of people with diabetes to 300 million by 2025 . According to a survey conducted by the International Diabetes Federation (IDF) already in 2013, 382 million patients worldwide have diabetes. Seven years later, in 2020, the number of people living with diabetes is 463 million.

There are two main types of diabetes: Type 1 diabetes can develop at any age, but is most common in children and adolescents. When you have type 1 diabetes, your body makes very little or no insulin, which means you need daily insulin injections to keep your blood glucose under control.

Type 2 diabetes is more common in adults and accounts for about $90 \%$ of all diabetes cases. When you have type 2 diabetes, your body does not use the insulin it makes well. Over time, most people with type 2 diabetes will need oral medications or insulin to keep their blood glucose levels under control.

In both of these cases, a person with diabetes needs to control their blood sugar levels to avoid complications of diabetes. Self-testing devices require a small amount of blood sampling using different needles, which causes pain and discomfort to the user of the device.

Today, a large number of methods and devices are being developed for determining glucose levels in a non-invasive and continuous method. This approach will allow people with diabetes to avoid the discomfort of measuring blood sugar and monitor them throughout the day.

The most famous non-invasive methods for measuring blood glucose are: Raman spectroscopy, impedance spectroscopy, near infrared spectroscopy, photoacoustic spectroscopy and others. 
Raman spectroscopy $[2,3]$ is based on the measurement of scattered light. The disadvantages of this method are the instability of the intensity and wavelength of laser beams during probing, a long time for obtaining data, as well as errors associated with chemical substances in the tissues. Impedance spectroscopy based on the measurement of resistance when the radiation frequency changes. To measure the glucose level, several sensors are needed located in the area of the veins in the human hands [4]. Near infrared spectroscopy is based on the transmission of near infrared radiation through the vascular region of the body (finger, earlobe, etc.). In this case, the glucose concentration is calculated on the basis of the received spectral information [5,6]. Mid-infrared spectroscopy is based on the absorption of light by glucose molecules [7-9]. This method uses a beam of light to travel through a crystal in contact with the skin, thus ensuring that the electromagnetic field generated by the reflected light reaches the dermis (the layer of skin that contains the most glucose). The disadvantage of this method is the dependence of the obtained data on the water content in the dermis. Therefore, the reliability of the results largely depends on the degree of hydration.

There is a technology based on ultrasonic sensing - photoacoustic spectroscopy [10,11]. This method is based on the acoustic response of an excited liquid using laser light. This method is similar to mid-infrared spectroscopy. And also other, less known methods for determining the level of glucose in the blood $[12,13]$.

Thus, the development of a non-invasive method for determining glucose remains an urgent task today. The modern method for the approach to creating a non-invasive sensor for measuring blood sugar described in this article is based on the use of near-field microscopy. The near-field method provides deep penetration of electromagnetic waves even in highly absorbing medium.

\section{Method}

\subsection{Hand Model}

In order to develop a new sensor for non-invasive diagnostics of blood glucose levels, it is necessary to take into account several factors that can influence the measured value of the reflected electromagnetic signal. It should also be borne in mind that the concentration of glucose in different tissues of a person is distributed differently, the thickness of tissue layers in different people is different, and these are previously unknown values for non-invasive measurements. When developing non-invasive methods for measuring glucose by electromagnetic methods, it is necessary to first of all consider the effect of skin layers (Figure 1).

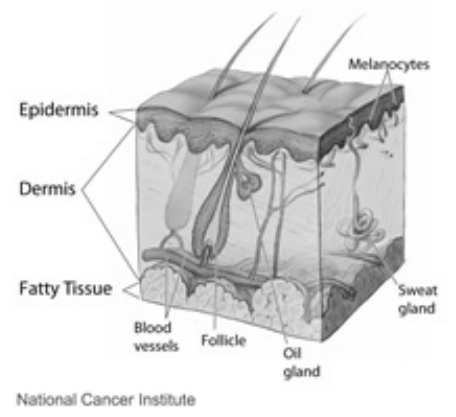

Figure 1. Skin structure.

This is important due to the fact that skin, like blood, has a high dielectric constant. Therefore, the electromagnetic wave attenuates in it more strongly than in other layers. Each layer of the skin has its own dielectric characteristics, which may differ from person to person due to differences in morphology and thickness of skin layers, concentration of tissue/blood components (such as glucose), cutaneous blood perfusion, etc.

The skin has a great influence on glucose measurement, which can vary in the range of 2-10 $\mathrm{mm}$ when looking at the whole human body. The skin has a multilayer structure (Figure 1) and 
includes: the stratum corneum - its thickness ranges from 15 to 150 microns in various parts of the body; epidermis - the upper outer layer of human skin, which includes about $15-35 \%$ of interstitial fluid, without blood vessels; the dermis, the skin itself, is a connective tissue: it contains arterioles, venules, capillaries and about $40 \%$ of interstitial fluid. Also, the dermis includes subcutaneous fatty tissue and loose fibrous connective tissue. The distribution and thickness of the skin depends on heredity, sex hormones and human conditions. On average, the thicknesses vary in the following ranges: epidermis thickness $-0.068-0.146 \mathrm{~mm}$; the stratum corneum of the epidermis is $0.021-0.049$ $\mathrm{mm}$, the dermis is $1.89-3.04 \mathrm{~mm}$, the subcutaneous fat is $0.03-1.41 \mathrm{~mm}$. The next layer is subcutaneous fat, which has a wide range of thickness. The thickness of the fat layer on the forearm is the smallest compared to other parts of the body.

It is also required to consider the saphenous veins running in the human forearm. To measure glucose with a standard glucometer, blood is drawn from the subcutaneous capillary vessels. In a medical examination, blood is drawn from a vein. The fundamental difference between these two methods is measurement accuracy. It should be understood that in medicine, the level of glucose (glycated hemoglobin) is determined in practice by venous blood. The content of these substances in venous and arterial blood is somewhat different. For a more accurate calculation of the concentration of glucose in the blood, it was the venous blood that was considered in our study. Vienna is a multi-layered structure. Depending on the type of vessels, they have different thickness, density and permeability. Large vessels additionally contain small blood and lymphatic capillaries.

Due to different pressures during the entire period of a person's life, the blood supply (blood volume in the measurement area) changes, along with this, the diameter of the vessels also changes. People with symptoms of tachycardia (increased heart rate) have smaller vessels due to the fact that with a rapid heartbeat, the efficiency of the heart decreases, since the ventricles do not have time to fill with blood. As a result, blood pressure decreases and blood flow to organs decreases, hence the smaller area for the microwave signal response.

Another factor that can affect the dielectric properties of blood is the blood hematocrit. Blood hematocrit refers to the percentage of red blood cells in the blood. Differences in the size, morphology and distribution of red blood cells in human blood lead to changes in its dielectric properties, regardless of glucose concentration and, thereby, affect the accuracy of glucose determination using measurement methods based on dielectric properties. Normally, this figure is $40-48 \%$ for men, $36-42 \%$ for women. At low frequencies (about $3 \mathrm{MHz}$ ), the spread in the dielectric constant is quite high in the region of $30 \%$ with a change in the hematocrit of 5\% [14]. This scatter is associated with a rapid change in blood dielectric constant from frequency, which is clearly shown in Figure 2. With increasing frequency, this effect disappears due to the linear behavior of the dependence of the real part of the dielectric constant. In this case, the change will vary by $0.01-0.02 \%$.

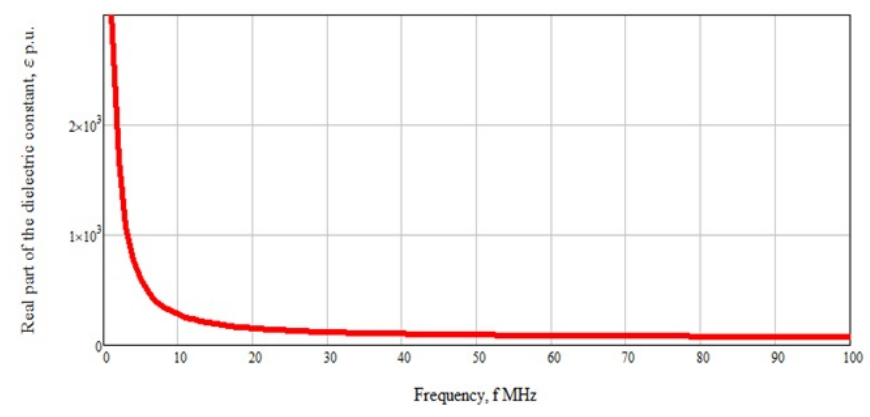

Figure 2. Dependence of the real part of the dielectric constant on frequency.

Based on the results of the literature review and analysis of the studied information, a summary Table 1 was formed with the thicknesses of all materials used for modeling. 
Table 1. Thickness of materials used in modeling.

\begin{tabular}{cc}
\hline Name of Materials & Thickness, $\mathbf{~ m m}$ \\
\hline Stratum corneum of the epidermis & 0.02 \\
Epidermis & 0.04 \\
Dermis & 1.83 \\
Subcutaneous adipose tissue & 1 \\
Vein hand & 4 \\
Hand vein wall & 0.5 \\
Fat & 6 \\
\hline
\end{tabular}

The values for the thicknesses of the muscles and bones of the human forearm weren't given in the table, since when the near field penetrates into these tissues, a strong signal attenuation occurs and the response from them will be at the noise level. Therefore, these layers in the calculation of the thicknesses of the materials used in the simulation can be neglected. Based on the given data, we have done the model of a biological medium sample in the form of a human hand (Figure 3).

This model has a flat-layered structure. All previously described materials were modeled and a $13 \mathrm{~mm}$ thick muscle layer was added. The use of a flat structure is due to the fact that the used antenna substrate is flexible. In the future, when creating a real prototype of the sensor, this will make it possible to attach it directly to the area of human skin.

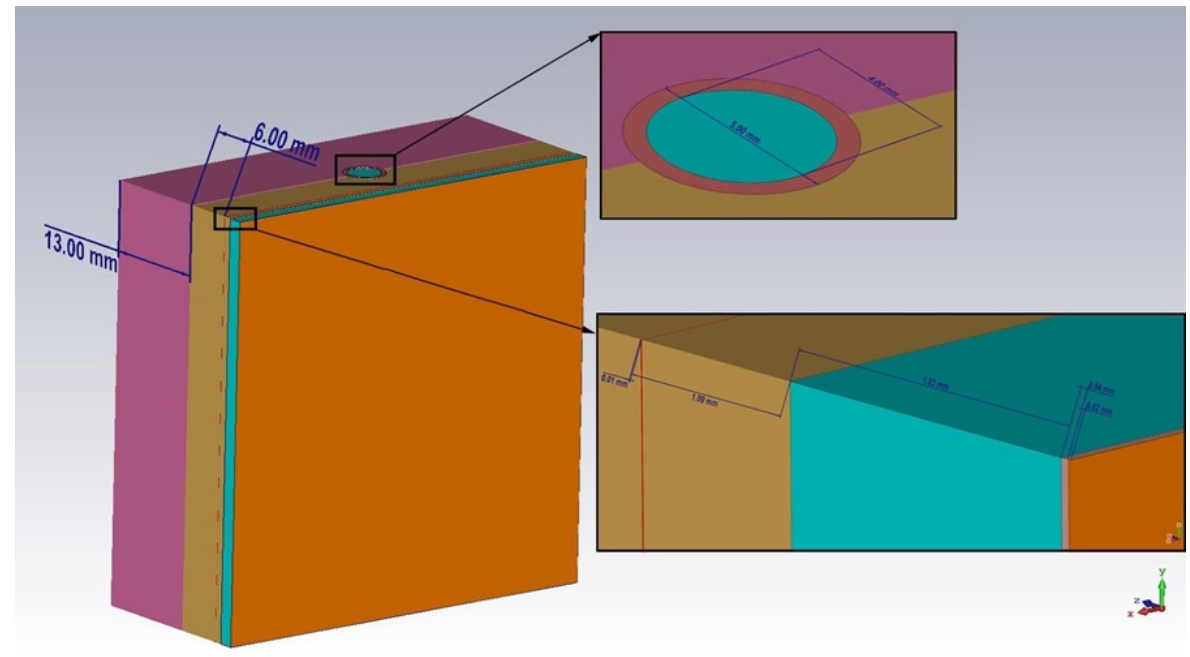

Figure 3. Model of the sample of the biological medium in the form of a human hand.

In the hand model, the data of the dielectric constant of saline with the following glucose concentrations were used as blood: 0, 1, 3, 4, 5, 7, 9, $10 \mathrm{mmol} / 1$ (Figure 4).

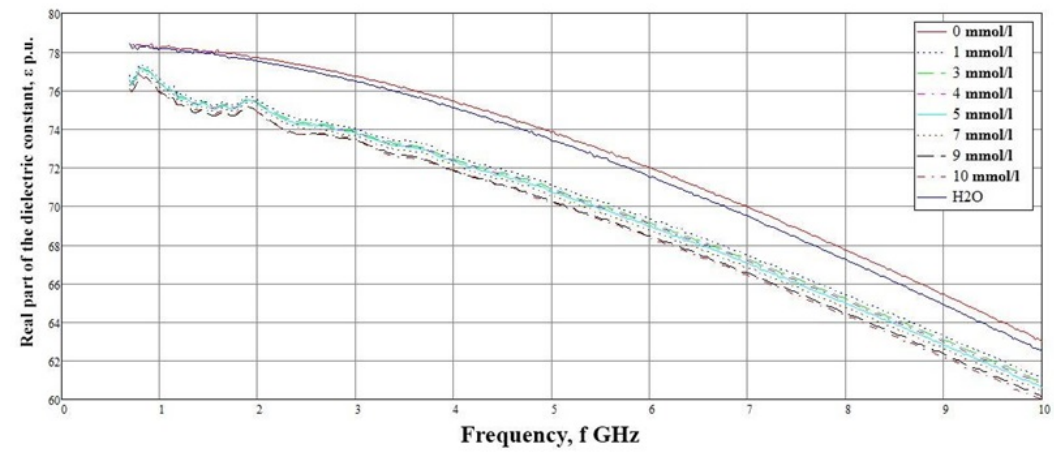

Figure 4. Model of the sample of the biological medium in the form of a human hand. 
This data was analyzed using a PNA-L Network Analyzer (N5230C) vector network analyzer and Dielectric Probe Kit - 85070E Slim Form from Agilent Technologies. In subsequent simulations for blood with varying glucose concentration, data obtained experimentally for physical solutions (saline) with different dextrose concentrations were used. Thus, it was possible to bring numerical modeling closer to real conditions.

\subsection{Sensor Design}

The development of the near-field sensor was based on a combined slot antenna. The brown color in the figure indicates the ideal conductor, green - the flexible dielectric RO3003 with relative permittivity $\varepsilon=3$. The shape of the sensor resembles a coin in the center of which there is a metallized circle with a slot made in it. Then, using two rectangular metallized sections, the circle is connected to a conductive frame (Figure 5a). The reverse side of the sensor is a dielectric layer with a microstrip line closed to a central circle, and a small cut in the dielectric up to the conductive frame - for connecting to the supply element (Figure 5b). The probing near-field is formed on the side with a slot (Figure 5a). Due to the presence of several radiating elements (slit and frame), the formation of reactive parts of a specific interference energy flow takes place. These features can increase the sensor's sensitivity for near-field diagnostics of biological media and objects.

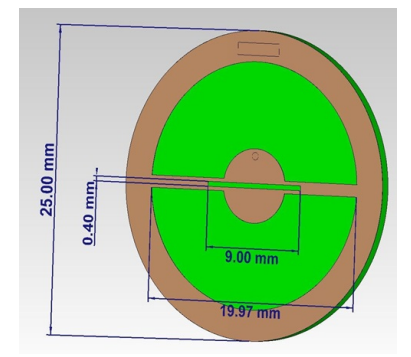

a)

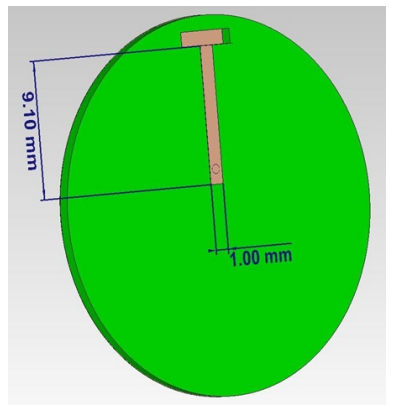

b)

Figure 5. Near-field sensor model.

The VSWR of the sensor is also considered in terms of coherence with a sample of a biological medium in the form of a model of a human hand (Figure 6). The graph shows two sections of the curve with the highest matching of the sensor and the sample under study: the first at a frequency of $1 \mathrm{GHz}(\mathrm{VSWR}=1.4)$, the second in the range of $2.1-5 \mathrm{GHz}(\mathrm{VSWR}=1.4)$. Note that almost the entire VSWR graph is below level 3.

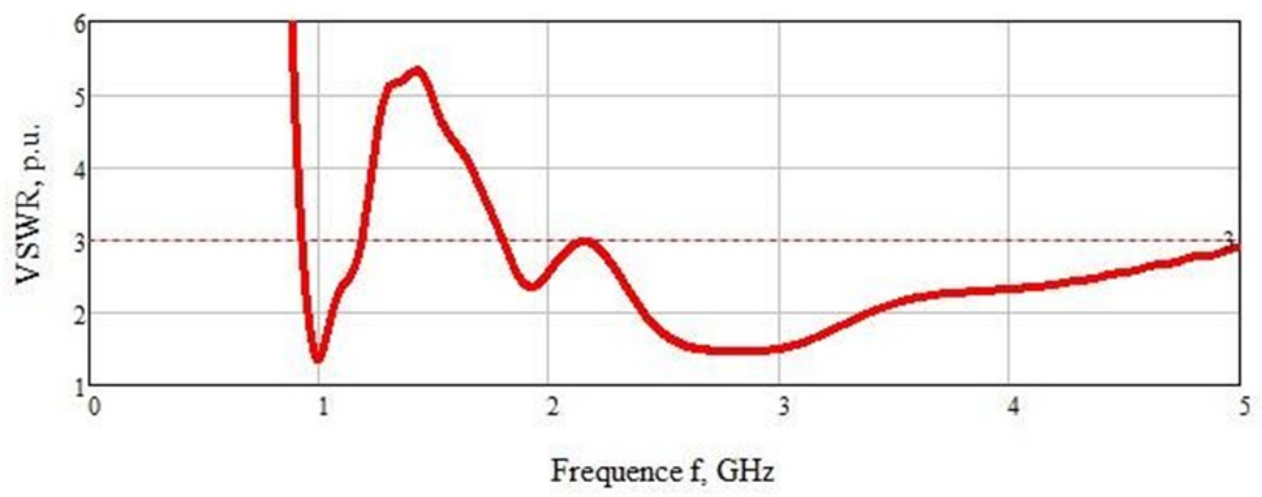

Figure 6. VSWR sensor coherence with the sample of the biological medium.

In the numerical simulation of the experiment to determine the concentration of glucose in the blood, the sensor was closely applied to a sample of a biological medium in the form of a model of a 
human hand. The measurements were carried out in the frequency range $0.5-5 \mathrm{GHz}$. We used this range to cover the entire frequency range in which the VSWR of the sensor is less than level 3.

\section{Results and Discussion}

The used sensor was located close to the hand model for maximum interaction of the near field with biological materials. The dependences of the reflected signal on the frequency were plotted (Figure 7a) as a result of a series of measurements for this model sensor. You may notice that the graphs are poorly distinguishable. It is due to small changes in the dielectric constant. When zooming in on a small area at $1 \mathrm{GHz}$, it can be seen that the most distinguishable reflected signal is observed for the hand model at $0 \mathrm{mmol} / \mathrm{l}$ blood glucose. The rest of the values differ only by a thousandth. So the values for the concentration of 1 and $3 \mathrm{mmol} / 1$ are -16.672 and $-16.671 \mathrm{~dB}$, respectively (Figure $7 \mathrm{~b}$ ). In this graph, it is not possible to visually determine the various concentrations.

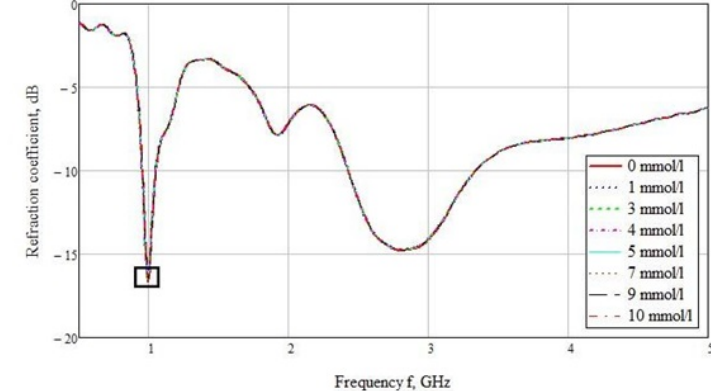

a)

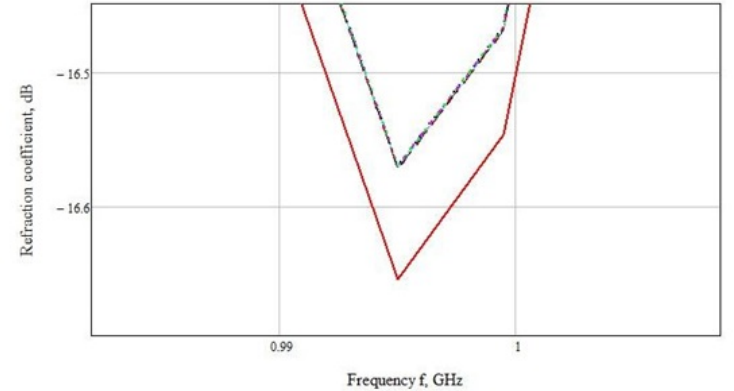

b)

Figure 7. Frequency dependence of the reflected signal for a hand model with different glucose concentrations.

For this, the subtraction of zero concentration was carried out according to the result of which the graphs presented in Figure 8 are obtained. Differences are visible at the amplitude peaks of $1 \mathrm{GHz}$ and the range of 1.5-1.8 GHz. Considering these graphs in more detail, we see a clear difference in concentrations at the frequencies presented above. To build a table with amplitude values, we use the maximum at $1.07 \mathrm{GHz}$.

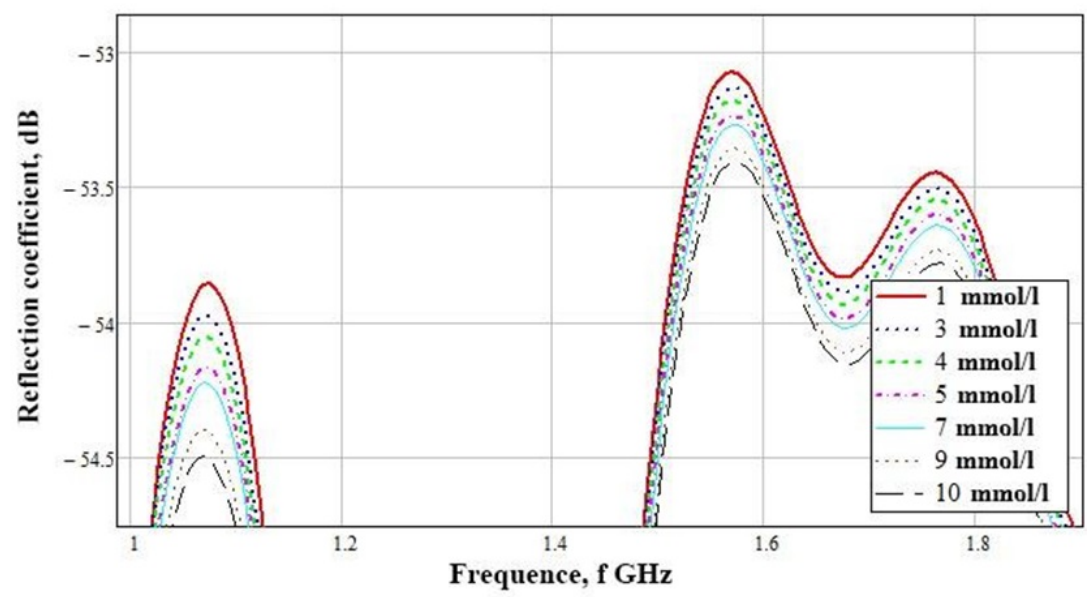

Figure 8. Parameter S11 for a model of a hand minus blood with a concentration of $0 \mathrm{mmol} / \mathrm{l}$.

Table 2 shows the numerical simulation data for a near-field sensor at a frequency of $1.07 \mathrm{GHz}$. This demonstrates its advantage in the accuracy of determining the concentration difference from the mean values. The difference of $1 \mathrm{mmol} / 1$ is $0.1 \mathrm{~dB}$. 
Table 2. The results of measuring the amplitude of the reflected signal by the proposed new sensor for different glucose content.

\begin{tabular}{ccc}
\hline Glucose Concentration, $\mathbf{m m o l} / \mathbf{1}$ & Frequency, $\mathbf{G H z}$ & Amplitude, $\mathbf{d B}$ \\
\hline 1 & 1.07 & -53.85 \\
3 & 1.07 & -53.97 \\
4 & 1.07 & -54.05 \\
5 & 1.07 & -54.16 \\
7 & 1.07 & -54.22 \\
9 & 1.07 & -54.39 \\
10 & 1.07 & -54.49 \\
\hline
\end{tabular}

As a result of theoretical studies and numerical modeling, a design of a new combined sensor based on a resonant antenna and a near-field effect was proposed to determine the concentration of glucose in a biological medium in the form of a model of a human hand.

\section{Conclusions}

In this work, a sensor based on a combined slot antenna was demonstrated. This sensor has an extended near field with a high penetration depth of the electromagnetic field. A study of the biological structure of the human hand in the elbow bend was carried out and subsequently a simplified numerical model was created. We also measured the reflected signal from the hand model with a shallow vein. The sensor gave a high echo response with a small change in the glucose level in saline.

The data obtained indicate the possibility of determining the co-concentration of glucose in the blood with great accuracy. The average scatter of the reflected signal data for a change of $1 \mathrm{mmol} / 1$ is $0.1-0.15 \mathrm{~dB}$, which is high for such a small change in glucose. Further research will be aimed at creating a prototype of this sensor and carrying out real experiments. The RO3003 backing is flexible enough to allow measurements to be made on different parts of the human body and to identify the most favorable location for its placement.

Author Contributions: Conceptualization, A.G., K.Z.; methodology, A.G., K.Z.; validation, A.G., A.M.; formal analysis, A.M.; investigation, A.M.; resources, A.G.; data curation, A.G., A.M.; writing-original draft preparation, A.G., K.Z., A.M.; writing-review and editing, A.G., K.Z.; visualization, A.M.; supervision, K.Z.; project administration, K.Z. All authors have read and agreed to the published version of the manuscript.

Funding: This research was funded by Russian Science Foundation, grant number 18-75-10101. The APC was funded by Russian Science Foundation.

Conflicts of Interest: The authors declare no conflict of interest. The funders had no role in the design of the study; in the collection, analyses, or interpretation of data; in the writing of the manuscript, or in the decision to publish the results.

\section{References}

1. Jones, M.; Harrison, J.M. The future of diabetes technologies and therapeutics. Diabetes Technol. $\mathcal{E}$ Ther. 2002, 4, 351-359.

2. Forst, T.; Caduff, A.; Talary, M.; Weder, M.; Brändle, M.; Kann, P.; Flacke, F.; Friedrich, C.; Pfützner, A. Impact of environmental temperature on skin thickness and microvascular blood flow in subjects with and without diabetes. Diabetes Technol. E Ther. 2006, 8, 94-101.

3. Hanlon, E.; Manoharan, R.; Koo, T.; Shafer, K.; Motz, J.; Fitzmaurice, M.; Kramer, J.; Itzkan, I.; Dasari, R.; Feld, M. Prospects for in vivo Raman spectroscopy. Phys. Med. Biol. 2000, 45, R1.

4. Caduff, A.; Hirt, E.; Feldman, Y.; Ali, Z.; Heinemann, L. First human experiments with a novel non-invasive, non-optical continuous glucose monitoring system. Biosens. Bioelectron. 2003, 19, 209-217.

5. Khalil, O.S. Spectroscopic and clinical aspects of noninvasive glucose measurements. Clin. Chem. 1999, 45, 165-177.

6. Heise, H. Non-invasive monitoring of metabolites using near infrared spectroscopy: State of the art. Hormone Metab. Res. 1996, 28, 527-534. 
7. Gebhart, S.; Faupel, M.; Fowler, R.; Kapsner, C.; Lincoln, D.; McGee, V.; Pasqua, J.; Steed, L.; Wangsness, M.; $\mathrm{Xu}, \mathrm{F}$; et al. Glucose sensing in transdermal body fluid collected under continuous vacuum pressure via micropores in the stratum corneum. Diabetes Technol. E Ther. 2003, 5, 159-166.

8. Lipson, J.; Bernhardt, J.; Block, U.; Freeman, W.R.; Hofmeister, R.; Hristakeva, M.; Lenosky, T.; McNamara, R.; Petrasek, D.; Veltkamp, D.; et al. Requirements for calibration in noninvasive glucose monitoring by Raman spectroscopy. JDTS 2009, doi:10.1177/193229680900300203.

9. Roychoudhury, P.; Harvey, L.M.; McNeil, B. At-line monitoring of ammonium, glucose, methyl oleate and biomass in a complex antibiotic fermentation process using attenuated total reflectance-mid-infrared (ATR-MIR) spectroscopy. Anal. Chim. Acta 2006, 561, 218-224.

10. Waynant, R.; Chenault, V. Overview of non-invasive fluid glucose measurement using optical techniques to maintain glucose control in diabetes mellitus. IEEE LEOS Newsl. 1998, 12, 3-6.

11. Khalil, O.S. Non-invasive glucose measurement technologies: An update from 1999 to the dawn of the new millennium. Diabetes Technol. \& Ther. 2004, 6, 660-697.

12. Gabbay, R.A.; Sivarajah, S. Optical coherence tomography-based continuous noninvasive glucose monitoring in patients with diabetes. Diabetes Technol. E Ther. 2008, 10, 188-193.

13. Guo, X.; Mandelis, A.; Zinman, B. Noninvasive glucose detection in human skin using wavelength modulated differential laser photothermal radiometry. Biomed. Opt. Express 2012, 3, 3012-3021.

14. Hayashi, Y.; Brun, M.A.; Machida, K.; Lee, S.; Murata, A.; Omori, S.; Uchiyama, H.; Inoue, Y.; Kudo, T.; Toyofuku, T.; et al. Simultaneous assessment of blood coagulation and hematocrit levels in dielectric blood coagulometry. Biorheology 2017, 54, 25-35.

(C) 2019 by the authors. Licensee MDPI, Basel, Switzerland. This article is an open access article distributed under the terms and conditions of the Creative Commons Attribution (CC BY) license (http:/ / creativecommons.org/licenses/by/4.0/). 